\title{
Concealed post-partum haemorrhageaemor: A case report
}

\author{
Anne De Haan, Noortje Van Oostrum, Page Geert Herman* \\ Department of Gynaecology and Obstetrics, Jan Yperman Hospital, Ieper, Belgium \\ Email: *geert.page@yperman.net
}

Received 19 January 2012; revised 20 February 2013; accepted 1 March 2013

Copyright (C) 2013 Anne De Haan et al. This is an open access article distributed under the Creative Commons Attribution License, which permits unrestricted use, distribution, and reproduction in any medium, provided the original work is properly cited.

\begin{abstract}
A woman with five previous normal vaginal deliveries and a history of right salpingectomy for ectopic pregnancy has sudden pain in term labour, with epidural analgesia. A concurrent worsening of fetal heart rate pattern with bradycardia urges quick vacuum delivery. Maternal post-partum pain and hemoglobin $(\mathrm{Hb})$ drop suggest intra-abdominal haemorrhage. The combination of sudden, strong intra-partum pain and fetal distress should raise the suspicion of intra-abdominal haemorrhage. After vaginal delivery an accurate follow-up is mandatory.
\end{abstract}

Keywords: Concealed Haemorrhage; Rupture of Utero-Ovarian Vessels; Labour

\section{BACKGROUND}

Although haemoperitoneum resulting from spontaneous rupture of utero-ovarian vessels during pregnancy and delivery is very rare, it is however a life-threatening complication for mother and child. Approximately 120 cases have been reported in literature, the majority $(61 \%)$ directly related to labour [1]. Maternal mortality rate has decreased significantly from $49 \%$ in 1950 [2] to $4 \%$ in 1987, mainly due to improvement in anesthetic and bloodbanking techniques. Perinatal mortality remains high at $31 \%[1]$.

Since presentation varies widely, diagnosis before surgery is notoriously difficult. Acute hypovolemic shock is the most typical finding. Other symptoms include abdominal pain and fetal distress.

The most important diagnostic distinction to make is the one between intra-abdominal bleeding and placental abruption.

\section{CASE REPORT}

A 32-year-old gravida 9 para 5, was admitted to the de-

\footnotetext{
"Corresponding author.
}

livery ward for induction of labour at her own request at 38 weeks gestation.

She had had 5 uneventful vaginal deliveries, 2 missed abortions and one ectopic pregnancy, treated by a right salpingectomy in 2011. In 2005 she opted for laparoscopic sterilization followed by laparotomic refertilization in 2009. Furthermore, in childhood, she underwent an uncomplicated appendectomy.

Induction of labour was performed through a combination of vaginal prostaglandin's and intravenous oxytocin. Fetal presentation was cephalic. Epidural anesthesia was installed at a dilatation of $4 \mathrm{~cm}$. Artificial rupture of membranes was performed, revealing clear amniotic fluid. An hour later the patient reported a sudden, sharp pain in the right lower quadrant. Dilatation at that time was $8-9 \mathrm{~cm}$. The pain remained, in moderate form, until delivery. Shortly after the commencement of pain fetal heart rate monitoring showed prolonged deceleration without recovery in between contractions. Meanwhile, the patient had reached full dilatation and a vacuum delivery was performed. Ten minutes after the start of fetal heart rate deceleration a male infant was born with Apgar scores of $2 / 4 / 8$, a pH of 6.84 and $\mathrm{BE}$ of -16 . The placenta and membranes followed immediately after. These showed macroscopic signs of haemorrhage, suggesting placental abruption. Pathology findings were not able to confirm this, though a small chorangioma was described. After delivery the uterus contracted well and blood loss was moderate. The previously described pain had eased and the patient was hemodynamically stable.

5 hours post partum, during the night, the patient felt faint and dizzy. When asked, she only indicated mild pain. At that time, her blood pressure was $60 / 40 \mathrm{mmHg}$, but recovered very rapidly after fluid loading. She was hemodynamically stable and conscious, but pale and clammy. Uterine tonus was normal as was vaginal blood loss. Ultrasound examination excluded intra-uterine retained tissue and intra-abdominal free fluid. Blood tests showed an $\mathrm{Hb}$ of $6.8 \mathrm{~g} / \mathrm{dl}(11.9$ - 14.7), a hematocrit (Hct) of $21.3 \%$ (33.6 - 44.0) and normal coagulation parame- 


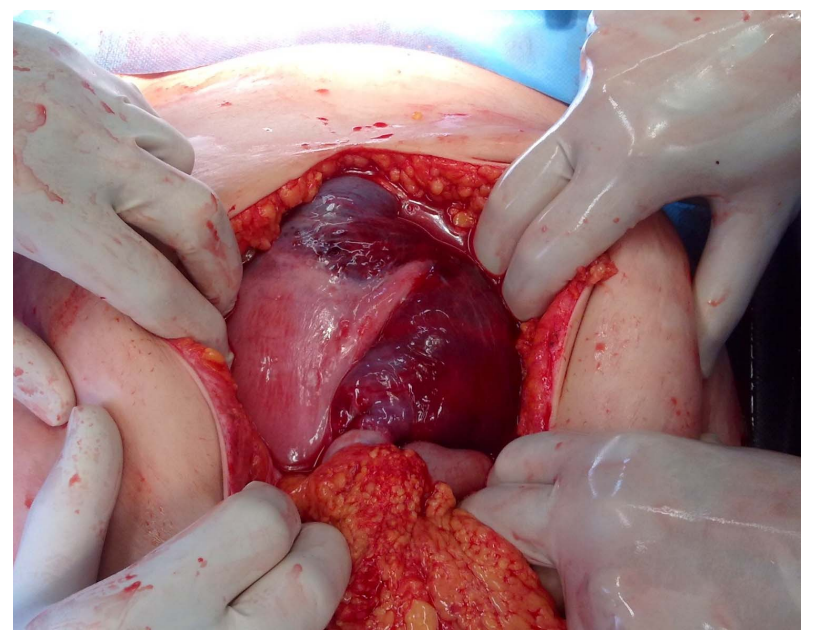

Figure 1. Exploratory laparotomy demonstrated a large retroperitoneal blood collection in the right broad ligament, extending to the entire right hemi-abdomen and to the right part of the vesico-uterine space.

ters. Patient was given 2 units of packed cells and 2 units of fresh frozen plasma based on the diagnosis of post partum blood loss. Intravenous oxytocin was restarted.

During the night the patient remained hemodynamically stable, but the abdominal pain worsened. Blood tests after transfusion showed an $\mathrm{Hb}$ of $6.0 \mathrm{~g} / \mathrm{dl}$ and a Hct of $18.6 \%$. A CT scan, performed in the morning, suggested intra-abdominal bleeding. Exploratory laparotomy was performed, illustrating a large retroperitoneal blood collection in the right broad ligament, extending to the entire right hemi-abdomen and to the right part of the vesico-uterine space. There were no signs of uterine rupture and no intra-abdominal free fluid was noted (Figure 1). The collection was left intact as it seemed to have compressed itself. A drain was placed in the pouch of Douglas. The patient was transfused once more and made good recovery within a few days.

A CT scan 6 weeks post partum showed a small residual collection, but the patient was clinically well.

\section{DISCUSSION}

The etiology of spontaneous rupture of a utero-ovarian vessels during pregnancy and labour is unclear. In $75 \%$ of published cases the broad ligament is the site of rupture [3]. The tortuous path and lack of valves of these vessels, combined with significant distension from elevated venous pressure in pregnancy and labour may predispose them to rupture. A possible individual underlying vascular defect may increase the risk [2].

Presenting symptoms vary widely. Acute hypovolemic shock and severe abdominal pain, resistant to epidural anesthesia, shortness of breath or shoulder pain may be noted [4-6]. As in this case, fetal distress may be present. These symptoms must create attentiveness of the health care provider and necessitate close monitoring.

The treatment should consist of aggressive replacement of fluids and blood to maintain adequate circulating intravascular volume. Rapid surgical intervention to definitively diagnose and if necessary stop intra-abdominal bleeding should be considered. Caesarian section should be performed if vaginal delivery is not imminent.

In the present case we found no definitive underlying cause for the bleeding. The blood collection was left intact as it had compressed itself. Therefore, the exact location of the rupture was not identified. However, no signs of uterine rupture were present because of which it can be assumed that the cause was more likely to be rupture of utero-ovarian vessels than uterine rupture.

The combination of severe abdominal pain and fetal distress must make the obstetric health care provider consider the possibility of a spontaneous rupture of the utero-ovarian vessels. Close monitoring of the patient and the fetus should be guaranteed and laparotomy to explore the abdominal cavity should be strongly considered.

\section{REFERENCES}

[1] Ginsburg, K.A., Valdes, C. and Schnider, G. (1987) Spontaneous utero-ovarian vessel rupture during pregnancy: Three case reports and a review of the literature. Obstetrics \& Gynecology, 69, 474-476.

[2] Hodgkinson, C.P. and Christensen, R.C. (1950) Hemorrhage from ruptured utero-ovarian veins during pregnancy: Report of 3 cases and review of literature. American Journal of Obstetrics \& Gynecology, 59, 1112-1117.

[3] Ziereisen, V., Bellens, B., Gérard, C. and Baeyens, L. (2003) Spontaneous rupture of utero-ovarian vesels in post partal period: A case report and review of the literature. Journal de Gynecologie Obstetrique et Biologie de la Reproduction, 32, 51-54.

[4] LaRose, P. and Sehdeva, P.K. (1978) Spontaneous rupture of a uterine vein during labor. Southern Medical Journal, 71, 1446-1447. doi:10.1097/00007611-197811000-00042

[5] Moreira, A., Reynolds, A., Baptista, P., Costa, A.R. and Bernardes, J. (2009) Case report: Intra-partum utero-ovarian vessels rupture. Archives of Gynecology and Obstetrics, 279, 583-585. doi:10.1007/s00404-008-0751-z

[6] Aziz, U., Kulkarni, A., Lazic, D. and Cullimore, J.E. (2004) Spontaneous rupture of the uterine vessels in pregnancy. Obstetrics \& Gynecology, 103, 1089-1091. doi:10.1097/01.AOG.0000121833.79081.c7 